the ERAS cohort and the legacy non-ERAS cohort (those who received the same types of surgery from the 2 ERAS attending surgeons prior to implementation of the protocols).

Results: A total of 244 patients were evaluated (122 patients in ERAS cohort vs. 122 patients in non-ERAS cohort). The number of vials of opioid analgesia used during the first 2 post-operative days were significantly lower in the ERAS group. The patients in ERAS group reported less post-operative pain, feelings of hunger and thirst, and greater amount of exercise post-operatively. These benefits of the ERAS cohort were more pronounced in the patients who underwent laparotomic surgeries than those who underwent laparoscopic surgeries. The case-matched comparative analysis also showed similar results. The length of hospital stay did not differ between the 2 groups.

Conclusion: The results of this study demonstrated the safety, clinical feasibility and benefits of the ERAS protocols for patients undergoing gynecologic surgeries.

Poster (M03)

Miscellaneous

https://doi.org/10.3802/jgo.2021.32.S1.MO3

\section{Clinical analysis of multiple primary malignancies in gynecologic cancer patients}

\author{
Eun Jung Yang, Yoo Jin Kim, A Jin Lee, Nae Ri Kim, \\ Seung Hyuk Shim, Sun Joo Lee, Tae Jin Kim, Kyeong A So* \\ Konkuk University School of Medicine, Seoul, Korea (20190001@kuh.ac.kr)
}

Objective: The aim of this study was to evaluate the prevalence and oncologic outcomes in multiple primary malignant tumors (MPMTs) with gynecologic cancers.

Methods: A retrospective study included 1,929 patients diagnosed with gynecologic malignancies at a tertiary institution medical center between August 2005 and April 2021. We analyzed MPMTs in gynecologic malignancies.

Results: The prevalence of MPMTs with gynecologic cancer patients was $8.6 \%(165 / 1,929)$. The median diagnosis period between primary and secondary cancer was 38 months (range 0-420). Among 165 MPMTs patients, 20 patients had multiple primary gynecologic cancers (MPGC), and 145 patients had gynecologic cancer coexisting with non-gynecologic cancer (GNC). The coexistence of endometrial cancer and ovarian cancer $(12 / 20,60 \%)$ was the most common in MPGC patients. The most common non-gynecological cancer in GNC patients was breast cancer (50/145, 34.5\%). Synchronous malignancies cases were 48 (29.1\%), and metachronous malignancies cases were 117 (70.9\%). MPGC patients had more synchronous cancer than GNC patients (50.0\% vs. $26.2 \%, \mathrm{p}=0.030)$. In patients with ovarian cancer, the diagnosis of early stage was significantly higher in MPGC patients than that in GNC patients (87.5\% vs. $52.4 \%, \mathrm{p}=0.031$ ). The mortality rate during study period was $7.3 \%$, all of which were GNC patients. Gynecologic cancers were a more direct cause of death than non-gynecological cancer (66.7\% vs. $33.3 \%)$.

Conclusion: Half of MPGC patients had synchronous cancer, which was significantly higher than GNC. Ovarian cancer showed a higher rate of being diagnosed at an early stage in MPGC patients than that in GCN patients. A systematic examination after the diagnosis of primary cancer can help to diagnose secondary primary malignancy at early stage and improve the prognosis of the patients.

Poster (M04)

Miscellaneous

https://doi.org/10.3802/jgo.2021.32.S1.M04

\section{Natural killer cell as prognostic factor in Thai gynecologic cancer patients}

\section{Suwanit Therasakvichya," Morakt Kanbun}

Faculty of Medicine, Siriraj Hospital, Mahidol University, Bangkok, Thailand (suwanit.the@mahidol.ac.th)

Objective: Natural killer (NK) cells are lymphocytes that are programmed in immune system to recognize altered/ or stressed by malignant transformation. In gynecologic cancers, the prognostic impacts of NK cell have also been published. Thus, this study aims to demonstrate a preliminary report of NK cell levels in correlation with treatment outcomes among Thai gynecologic cancer patients. Methods: This cohort comparative study was conducted on 122 women with gynecologic cancer who have been treated with chemotherapy at Siriraj Hospital, between March 2011 and March 2012. The assessment of NK cell using flow cytometry (FACscan) with CD16/CD56 surface markers was done prior to the first course of chemotherapy, which reported in the percentage of NK cells and NK cell count. The majority chemotherapy was carboplatin and paclitaxel (PT) regimen every 3 week. The patients were divided into 2 groups based on the outcomes of the treatment: responder group (complete response or partial response), and non-responder group (stable disease, progression of disease, death with disease). Results: After completion of 6 cycle-chemotherapy, the median follow-up time was 7.3 months (range 1-18 months). The mean percentage of NK cell in responder and non-responder group were $19.5 \% \pm 9 \%$ and $19.6 \% \pm 8 \%$, respectively $(\mathrm{p}=0.949)$. The median NK cell count in responder group was 313 cells/ $\mu \mathrm{L}$ (range 95-1,255) compared to 282 cells $/ \mu \mathrm{L}$ (range 66-1,885) in non-responder group $(\mathrm{p}=0.276)$. The mean of NK cell count in patients with death with disease, alive with disease, and alive without disease were $171 \pm 95$, 